\title{
Impennarsi ed altre voci affini.
}

Donde l'impennarsi o impennare del cavallo? I vocabolarii della lingua lo imbrancano con l'impennare o impennarsi che significa, in senso proprio o metaforico, vestir di penne, metter le penne, o metter in carta (l'opposto di depennare), e altre cose simili evidentemente ricollegantisi a penna, sia nel valore suo più generico, sia in quello specifico di 'penna da scrivere'. Quei lessici dicono o lasciano intendere che, quando il cavallo alza le gambe davanti, par come se si levasse a volo. Eppure un tal traslato, per quanto non addirittura impossibile, giacche le lingue ne fanno di ben più arditi o assurdi, non mi è mai parso verosimile. Il quadrupede, quando s' impenna, allora è più che mai remoto dall' arieggiare il volo, poichè stenta a pur camminare. Quel che invece è caratteristico e appariscente è che allora, se perde in mobilità, guadagna in altezza; e ciò è giusto messo in rilievo dal sinonimo inalberarsi.

C' è ben di più. Lo spagnuolo ha il verbo empinar, che è stato pur esso poco fortunato in mano agli etimologi. Significa 'alzare, ergere, drizzare in alto', e s' adoprò anche per l' innalzamento d' una persona ad un alto grado, qual sinonimo di encumbrar *inculminare; ed empinarse si dice cosi dei quadrupedi che s' impennano, come degli uomini che s'alzano in punta di piedi, e come di alberi o torri o montagne che eccellano sopra quanto le circonda. Se il verbo italiano puó anche prendere il senso di 'scattar sù impermalito', quel dell' Iberia può prender il senso di 'inorgoglirsi', come pur fa il nostro inalberarsi. Per dipiù, empinar neutralmente vale altresì 'bever molto'.

Veramente, quanto a quest' ultimo significato, sembrò al Diez che se n' avesse a fare una cosa a parte, considerandolo come un grecismo e confrontandolo col pyer d' un vecchio testo francese, che ritenne scherzosamente foggiato sull' infinito aoristo $\pi \iota \varepsilon \tau \nu .1$ Non mi è ben chiaro se il Diez intendesse riconoscervi una formazione grecanica che sol per caso venisse a suonar identica all' empinar 'alzare', o se invece vi scorgesse una scherzevole applicazion di quest' ultimo al 'bere' suscitata dalla fortuita sua omo-

1 Etym. Wörterb. der roman. Spr. II c., s. pier. 
fonia con la voce greca. Certo che nell' utilissimo libro del Körting si trova ora impostato un $\varepsilon \mu \pi \tilde{\imath} \nu \varepsilon \iota \nu$, qual possibile ètimo del verbo spagnuolo in quanto odora di vino. 1 Sennonchè codesto senso speciale, che il Dizionario dell' Accademia madrilena dà per familiare e per antiquato, non deve staccarsi nè punto nè poco dal resto; e si ridurrà a una mera ellissi, come fa intravedere, per esempio, questo luogo del Don Chisciotte (I, cap. VIII), ove Sancio "de cuando en cuando empinaba la bota [ 1 ' otre] con tanto gusto que le pudiera envidiar el mas regalado bodegonero de Málaga ". Del rimanente, al nostro alzare il gomito, al francese hausser le conde, lo spagnuolo risponde con alzar o empinar o levantar el codo o de codo; dove è lecito notare che questa stessa oscillazione tra il complemento diretto (el codo) e l' indiretto (de codo) che sa di modo avverbiale, doveva agevolar l' ellissi, e condurre a poter dire bonariamente empinar senz' altro. Se ciò ebbe luogo solo con quest' uno dei tre verbi sinonimi, può esser caso, e può anch' essere effetto dell' idea di 'esaltazione', nel senso cattivo come nel buono, che in empinarse campeggia; nè sarà inutile ad ogni modo il richiamare il nostro altelto o alticcio per 'brillo'. Già il Liebrecht, come apprendo dal Körting, mise in rilievo che il verbo per 'bere' ¿̀ insomma il medesimo 'alzare', benchè, a quanto sembra, senza ch' egli riuscisse a indicare chiaramente la base latina su cui stia l'empinar tutto quanto.

Ebbene, qui e il Diez stesso (Il b, s. pino) e il Körting (n. 478I) pensano a un *impinare, che primamente importasse 'drizzar sù come un pino'. L' it. inalberarsi, e lo sp. arbolarse o enarbolarse, che son pretti sinonimi di impennarsi e empinarse, e il tedesco sich bäumen, sembrano a ciò un ovvio rincalzo; e quantunque l' albero avuto qui in mira dall' intuitiva dei parlanti sia stato (cfr. Diez, I, s. alberare) quel della nave, non perciò ci dilungheremmo dal 'pino', che per tanti secoli fu l' albero marinaresco per eccellenza. Però, se così venne il suo nome ad usarsi per la 'nave' stessa, non trovo che si usasse mai per 'albero della nave'; nè d' altra parte mi par molto probabile che l' intuitiva popolare $s$ ' inducesse a mettere al posto dell' idea di albero, comunque inteso, l' idea particolare del pino. S' aggiunge che il portoghese chiama pinho quel che l' italiano e lo spagnuolo pino, e tuttavia dice pur esso empinar e empinarse in tutti i significati della lingua sorella, senza che si abbia ragione alcuna di sospettare che ciò sia per imprestito dallo spagnuolo. Finalmente, il nostro impennare, che assolutamente non può staccarsi dal corrispondente spagnuolo, non può aver nulla da spartire col pino. Come viceversa empinar nulla può aver da fare con penna. Malgrado la timida apparizione degli ormai antiquati pena 'penna' e 'pelliccia', peña 'pelliccia', e pénola o péndola 'penna' pennula, il vero termine spagnuolo è pluma (quindi emplumar ed emplumecer), più ancora risoluta-

1 Lateinisch-romanisches Würtcrb., Paderborn I90I, num. 3243. 
mente che in francese. $E$ dal canto suo il portoghese, che s' avvicina all' italiano nella predilezione per penna, dice empennar nel senso che si attiene alle ali, di contro a empinar alzare; e così ribadisce la necessità, che fin dal principio abbiamo affermata, di tenere in italiano ben distinti tra loro $\mathrm{i}$ due diversi impennare.

Tutto si schiarisce se ci volgiamo a pinna. Il suo senso fondamentale era di cosa acuta o sporgente o laminare, donde le varie accezioni: 'punta della penna o dell' ala o della coda, cresta del gallo, pinne dei pesci, merli del muro, specie di conchiglia', e via via. L' estrema somiglianza di suono con penna (l'organo del volo: cfr. $\pi \dot{\varepsilon} \tau-o \mu \alpha \iota$ ), la rassomiglianza generica del concetto, per esser anche l' ala un che di sporgente, e i contatti specifici in casi particolari, per aver anche la penna una punta, e la possibilità di un maggior equivoco in qualche parola composta, condussero presto $i$ latini a uggiose confusioni, che $i$ grammatici si sforzavano di dissipare. Si dovè avere dapprima, nettamente separati, un aggettivo bipinnis, 'a doppio taglio' (quindi sostantivato 'scure'), e un bipennis, 'a due ali', il qual senso ricorre certamente in Plinio riferito agl' insetti. Ma già ai tempi di Quintiliano era tanto invalso di chiamar bipennis la scure, che egli s' affannava a inculcare come tal composto sia da pinna, non già da penna, secondo taluni credevano, e che inoltre non se ne debba quindi argomentare, come taluni facevano, che si possano dir pinnas anche le penne dell' uccello. 1 E Flavio Capro, un grammatico dei tempi di Traiano, ammoniva: "pinnas murorum, pennas avium dicimus". Dal luogo di Quintiliano apparirebbe che il bipennis fosse uno dei primi incentivi alla confusione tra i due sostantivi, e che ad ogni modo soprattutto per esso i nodi venivano al pettine. Isidoro se ne giovava ad argomentarne che penna fosse stato l' originario suono del vocabolo, e pinna fosse una posteriore alterazione. La scure, dice lui, in antico si diceva penna, e, se a due tagli, bipennis, e pennum dicevan gli antichi ciò che $\dot{e}$ acuto; onde son pur le pennae degli uccelli, perchè acute; e questo è il nome che conserva la vera forma antica, giacchè gli antichi dicevano appunto pennas, non pinnas. ${ }^{2} F_{u}$ scritto che qui Isidoro confonda, per il primo, interamente i due nomi; ma credo bisogni bene intendersi. Che egli ne faccia tutt' uno etimologicamente parlando, è manifesto; come è pur evidente che secondo lui la forma pinna non ha

1 Quare discat puer, quid in litteris proprium, quid commune, quae cum quibus cognatio; nec miretur cur ex scamno fiat scabellum, aut a pinna (quod est acutum) securis, utrinque habens aciem, bipennis: ne illorum sequatur errorem, qui, quia a pennis duabus hoc esse nomen existimant, pinnas avium dici volunt $(\mathrm{I}, 4, \mathrm{I} 2)$.

2 Securis apud Veteres penna vocabatur; utrinque autem habens aciem, bipennis. Nam bipennis dicitur quod ex utraque parte habeat acutum, quasi duas pennas. Pennum autem antiqui acutum dicebant. Unde et avium pennae quia acutae. Et ecce nomen quod reservavit antiquitatem, quia Veteres dicebant pennas, non pinnas (XIX, 19, 11 ). 
nulla di primitivo, è una posteriore alterazione di penna. Ma non è meno evidente che nell' uso comune a lui noto la distinzione si faceva ancora abbastanza tra le pennas degli uccelli e le pinnas dei muri; altrimenti non esclamerebbe, con tanta gioia, che nel primo termine è bellamente conservata la forma antica (antiquitatem) del vocabolo originariamente unico. Vale a dire che il secondo termine, a parer suo, degenere, era quello indicante le pinne dei muri. Il suo modo d' esprimersi è spiccio, epperò atto a indurre in errore un lettore moderno, il quale alla lesta può giudicare che, come i manoscritti latini di frequente confondono le due forme, così Isidoro non sia più in grado di discernerle affatto. No, e ci preme insistervi, tanto più che si tratta d' uno spagnuolo: cadde in un abbaglio etimologico, (anc' oggi c' è chi vi cade!), ma appunto con l' importanza di cimelio fonetico ch' egli attribuisce alla penna dei volatili, dà a divedere come non fosse allora tramontata la sua distinzione dalla pinna dei muri. Sussistevano insomma nella tradizione letteraria due forme e due significati, in continua attrazione reciproca, e ciò portava a confondere, $o$ in senso storico $o$ in senso pratico, or le forme or i significati, or quelle e questi insieme, donde una perplessità grande nelle scuole e tra gli amanuensi, la quale ha $i$ suoi ultimi echi fin tra $i$ latinisti moderni; ma la tenace vitalità delle due parole e lo zelo dei dotti, ancorchè talvolta ei non vedesser chiaro che a mezzo, impedirono un totale smarrimento.

Nel latino parlato si ebbero vicende non molto dissimili. In Italia la penna fu interamente attratta, in quanto al suono, nell'orbita della pinna: chè troviamo penna con e stretta nel toscano e in tutte le loquele che vanno con esso, non già aperta come l'e breve latina esigerebbe; e cosl in siciliano, pinna, non penna; e cosl in sardo, pinna, non già penna, come un' e latina, fosse pur lunga, avrebbe dato. Fui, credo, il primo ad asseverar tutto ciò, 1 sebbene con schiarimenti che oggimai mi sembrano non abbastanza precisi, e con riferimenti alla presunta origine del latino pinna, nella quale è meglio non addentrarsi, come cosa ch' è alquanto dubbia 2 e punto necessaria all' indagine romanza. Tuttavia la genuina penna,

1 Nel Grundrifs d. roman. Philol., I, 503.

2 Un problema mi pare, in che rapporto stia il termine latino, di cosi largo e fertile significato, col greco $\pi i v v \alpha \pi l v v \eta \pi \tilde{\imath} \nu \alpha$, che ha l' unico senso di 'conchiglia', con gli altri che ne dipendono, cioè un solo dei tanti che il latino abbraccia. Nè la voce greca è recente, poichè occorre già nella commedia attica, ed ebbe $i$ suoi riverberi pur nel lessico latino, grazie ai composti pinnophylax e pinnoteres o pinnotheras. Il termine greco e il latino risalgono alla solita fonte comune? o il latino è un antico grecismo divenuto qua prolifico di nuovi sensi e di proprii derivati ( $p$ in naculum ecc.)?! o il greco è un vecchio latinismo magnogreco propagatosi fra le ciurme dell' Ellade? Certo che questa specie di conchiglia, come mi fa notare il collega Kerbaker, ha forma così particolare, già descritta da Aristotele, che ne risulta non casuale la sua omonimia con la pinna significante 'quidquid est acutum'. 
continuante la vera pinna dell' età classica, si scova ancora nel lessico italiano, benchè appiattata nei vocabolarii tra le varietà ideologiche dell' usuale penna che è tralignamento della classica penna. 'Sopra le penne del monti' si legge nel Buti; 'penne dell' Alpi' nel Giambullari; 'penna d' un promontorio' nel Buonarroti. ${ }^{1}$ Nelle penne dell' istrice, pungiglioni ciod o peli spinosi, sarebbe lecito ravvisar nient' altro che 'punte', se non fosse legittimo il dubbio che possa ridursi a un mero traslato delle penne solite. La penna del martello, cioè la parte spaccata, che si contrappone alla bocca, sembra per la sua forma laminare includer l' idea di 'pinne', sebbene per la sua spaccatura ha richiamato a qualcuno la penna da scrivere, e sebbene il corrispondente francese penne abbia dato luogo ad altre supposizioni. ${ }^{2}$ La penna dell' antenna, cioè la sua parte più lunga e sottile, opposta al carro, la quale si manda in alto, e che diè luogo alla frase far la penna (mandare in alto l' antenna) e far 'l uomo alla penna (legarlo alla penna e alzarlo quanto più si può in alto a veder il mare), sarà reliquia della voce nel senso di cima o punta. ${ }^{3}$ Non parliamo delle penne nella cocca della freccia, poichè son piume davvero e in ogni caso potrebbero aver aria di ali onde par che la freccia voli; 4 nè della penna del fegato (par che viva specialmente nel bolognese e nel siciliano), che è difficile decidere che non sia un pretto sinonimo del solito ala di fegato, pur essendo più probabile che sia il caso inverso a quel di ailes du nez e ali del naso in cambio del dotto it. pinne, o di ali o alette dei pesci per pinne. Più importa il comasco 'pena della zappa', cioè il taglio; mentre poco rileva il penna $d$ ' apis che c' d dato per 'punta di matita' dal bergamasco, e invece per 'asta' dal veneziano, giacchè non solo tal discrepanza dà sospetto di poca precisione in uno dei due lessicografi, ma c 'è di mezzo la troppa affinità con la penna da scrivere.

Più copiosa e più sicura messe dà la toponomastica, in ispecie più sù $\mathrm{e}$ più giù del Tronto. Lascio che a Trivento (Molise) si chiama la penna la ripida pendice del monte al di là del Trigno, e chi sa in quanti altri posti si troverebbero simili appellazioni affatto paesane, non giunte mai agli onori dei vocabolarii geografici, bensì soltanto a quelli degli atti notarili o catastali; e pi-

1 Ho sorvolato su penna dello scudo nell' Ariosto (XII, 83, I), perchè pene de " escut nel Roland e in altri antichi testi oitanici (cfr. Godefroy) non è ben chiaro, e $i$ dotti inclinano a riconoscervi il cuoio o in genere la copertura dello scudo.

$2 \mathrm{Fu}$ raccostato al ted. (Hammer)bahn (Littré), ma, se non erro, questo è la 'bocca' del martello, ed è (Hammer)pinne la 'penna' (cfr. anche Scheler); e il Pinne e Finne delle favelle germaniche si riconnette a pinna.

3 Nel fr. penne (de l'antenne) il Littré ravvisa lo stesso basso brettone pen, estremità, testa, che egli reputa base dell' altro penne (che fu scritto anche pienne) significante nel gergo dei tessitori 'testa della catena'. Ma già il Diez notava che l' etimo brettone avrebbe sicuramente dato un maschile.

- Cfr. fr. penne e pennon (arc. penon). 
gliamo quei vocabolarii. Oltre Penne (Pinna), l' antica città vestina dei Pinnenses, abbiamo nello stesso Abruzzo Teramano Penna e Pennasantandrea; nel Chietino Pennadomo (pinna domus), Pennapiedimonte, Punta della Penna; presso Terni Penna in Teverina; nella provincia di Macerata Penna San Giovanni; in quella d' Urbino Pennabilli; nell' Appennino umbro marchigiano il Monte della Penna; nel ligure il Monte Penna; e al nord di Brindisi il Faro Penne (capo Gallo). Nel:' Appennino sannitico pure questi monti: Penna, Pennone, Pennapizzuto; ed uno dei più alti comuni del Molise è Pesco Pennataro, ove già Pesco indica l' altitudine montuosa, ma l' aggiunta può ben ribadire il medesimo concetto. 1 Schivo di frugare dove si rischia di dar di cozzo nelle Alpi Pennine o in un quissimile, ovvero in derivati vernacoli del verbo pendere. ${ }^{2}$ Ricordo invece quel che raggranello il Pieri nelle sue felici esplorazioni toponomastiche per la valle del Serchio e della Lima. ${ }^{3}$ Notato che nel territorio lucchese il vocabolo si vede applicato a fianco di monte o di colle che venga giù a picco, raccoglie più d' un Penna o Penne, talora preceduto da Alla o Alle; e Pennammuro (p. a perpendicolo); Alla Pénnora (pinnula); Pénnori, Trapénnori, Pennarella, Pennácchiore, Soppenna, Suppinnula, Soppennori. Tali riscontri fra contrade cosi discoste come Abruzzo e Lucca, lasciano intravedere quanto ci sarebbe forse da spigolar altrove.

Nelia Francia meridionale, se non altro quattro comunelli portano il nome di Penne, di La Penne, di Les Pennes. ${ }^{4}$ Ma la

1 Il che fu già intuito da G. Grasso in una delle sue ottime Note glottologico-geografiche (Rendiconti del R. Istituto Lombardo, serie II, vol. XXXII, a. 1899, p. 12 dell' estr.). Solamente, poichè non è addirittura un Pesco-Penna, lo sviluppo formativo nel secondo elemento apre la via a sospettar che in quest' ultimo non fosse piuttosto l' accenno a mura coronanti la rupe (cfr. più in là il sic. pinnata). I due Pignataro della Campania non aiutano a risolvere il piccolo dubbio, e per poco non tentano a fantasticare se il paesello sannitico non fosse in origine pur esso da pigna (pino), che ha dato qualche Pignato o Pignatella velletrano (Crocioni) e il Pignati (plur.), Pignatu, Pignatuni, Pignataru, di Sicilia (Avolio). Comunque, il nome campano e il siculo ci aiutano pel suffisso. Quanto a Pesco, già rilevò l' Ascoli (A rch. Gl. IIl $459 \mathrm{sgg}$.) la sua cognazione con pensilis, e le indagini realistiche del Grasso non han fatto che arrecarvi conferma; sennonche io porrei francamente * pensulus (contaminazione di pensilis con pendulus), donde pésolo e pènzolo e b. lat. pes(u)lo con pesclo ecc. (cfr. Arch. Gl. XIII, 4I 2).

2 Qui spettano i varii Pennini e il Pendino, e il vico Penninata di Napoli, e la Pennina di Campobasso: strade o rioni scoscesi; e potrebbe spettarvi anche il Monte Pennino dell' Appennino umbro-marchigiano; e certo il Pinninu, -eddu, -eddi, e il Pinnenti (pendente), dàtici dall' Avolio nel suo eccellente Saggio (Arch. Gl. Suppl., VI, p. 89). Al 'pino' invece collega egli Pinnolu (83) e Pinnatazza (ib.) e considera arabico Pinnicchi (82). Non so che pensare di Pinnazzi (Salerno); o del Pinadengo leventino (da pino?) di cui il Salvioni, Dei nomi locali leventinesi in éngo ecc., Bellinzona 1899 , p. 5. Ancora a pendere risaliranno Monte Pénnolo (Crocioni, La toponomastica di Velletri, Roma 1901, p. 20), e il Pennino Pennicello di cui il Rolla, Toponimia spicciola, Nicosia 1898 , p. 15-16.

8 Arch. Gl. Suppl. V, r6c.

- Il Ducange richiama penne e pennette, ai quali non trovo conferma negli altri libri che ho sotto mano. Il Godefroy ha penne, oltre pinnes, per 
vera miniera è l' Iberia, dove lo sp. peria e pg. penha e cat. penya è il termine usuale per „rupe, roccia, pietra viva “, e ha dato luogo agli usuali derivati peñasco o peñado, e peñascal, peñascoso (pg. penhasco, penhascoso); come ha dato luogo a un gran numero di nomi geografici, applicati a comuni, a villaggi, a monti, a promontorii, a contrade: i quali trovano eco pur nel Nuovo Mondo. Trascegliamo Peña, Peña de Francia, Sierra de la Peña, Peñafiel e Penhafiel, Peñafor, Peñalba, Peñamellera, Peñanigra, Peñas de san Pedro.

Coi nomi locali abbiamo già anticipato qualcosa sulla serie dei derivativi e dei composti, alla quale ora ci volgiamo. Una base *pinn-io fu già postulata dal Diez e dal Gröber per pignone e fr. pignon. Il primo è una specie d'argine fluviale $e$ ha dato il nome ad un luogo prossimo a Firenze nel quale l' Arno strariperebbe se non avesse quel riparo, e si trova anche per 'bica' (i pignoni dell' orzo). Il secondo (b. lat. pignio in Ducange) è 'muro che termina in punta e regge il colmo del tetto' (quindi la frase avoir pignon sur rue avere una casa propria), e nel gergo dell' orologeria è una piccola ruota dentata. L' antichita di alcuni esempii italiani, e le applicazioni affatto diverse, tra le due lingue, dell' identico concetto fondamentale, escludono il sospetto del francesismo fra noi. Bene invece sarà francesimo lo sp. piñon, limitato com 'è al senso dell' orologeria, e che non è da confondere con l' altro piñon, pg. pinhão, fr. pignon, pinocchio o pignuolo, risalente a pinea; il quale però potrebb' esser forse pur esso, in quanto è delle Spagne, un gallicismo, vista l' accezione diminutiva del suffisso, propria del dominio francese. Al proposito farebbe piuttosto lo sp. peñon, grossa rupe o masso di rupi, se la solita funzione accrescitiva non fosse indizio di formazione secondaria e più recente, da ridursi a un semplice ${ }^{*}$ pinn-one.

Difficile dire se qui giovi non dimenticare in tutto il pennone, fr. pennon, prov. peno, cat. pano, sp. pendon, pg. penas, pendão. Il Diez fra $i$ tre etimi che gli parevan possibili, pannus, pendere, penna, preferiva il terzo, e gilistamente avvertiva che, mentre non sarebbe troppo verosimile la riduzione di $n d$ in $n n$ nel francese e italiano, la degenerazione inversa sia facile nello spagnuolo, dove gli bastò addurre il péndola pennula, da noi dianzi registrato. Sennonchè sarebbe pur lecito dubitare se non quadrasse meglio pinna, trattandosi soprattutto di banderuola in punta alla lancia. Che pennoncello occorra talvolta per 'pennacchio sull' elmo' importa poco, essendo casi rari, relativamente tardivi, di applicazione evidente-

le alette dei pesci. Il Littré dà un panne (p. 4 ), pezzo di legno nella copertura d' una casa sotto $i$ tegoli, e un altro $(p .5)$ che nella Francia del Nord indica una specie di tegoli. Ei ne fa un femminile di pan pannus, ma si può dubitare, benchè pan abbia il senso di muro e d'altro; anzi di quest' ultimo si può sospettare che sia pinna mascolinizzato. Di panne du marteau abbiam gia discorso. 
mente secondaria. Con l' idea fondamentale e col significato più ordinario nulla han da fare le penne, se non le invochiamo in via di paragone o d' immagine; laddove la punta, a cui la banderuola si attacca, v' entrerebbe direttamente. Soltanto, qual rapporto ideale indicherebbe qui il suffisso? Chiameremmo noi 'puntone' o 'puntina' la banderuola? $E$ del resto, qual fondamento ha poi la generale presunzione che codesto vocabolo appartenga al fondo comune neolatino? Lo sp. pendon sarebbe sempre, nella migliore ipotesi, un termine semidotto (non essendo *peñon), e sard̀ più verosimilmente un gallicismo; con che si spiega anche meglio l' ingenuo raccostamento a pender, e l'oscillazione del portoghese tra una forma più prossima al francese, che già scrisse penon, e la forma spagnoleggiante. Circa l' italiano torna superfluo richiamare quanto ei fosse tributario alla Francia per quel che s' attiene alle cose cavalleresche, e quanto sia legittimo qui, malgrado il nessun indizio fonologico, intravedere il gallicismo. $\mathrm{Ma}$ se così è, se tutto si riduce a scrutar la formazione del solo termine francese, allora non solamente vacilla sempre più il mio pinna, ma si può finanche ridar lena al pannus, che il Littré non osava difendere più energicamente contro il Diez per mero riguardo al riflesso italospagnuolo; 1 e alla banderuola converrà forse dipiù l' idea di 'pannuccio' che non quella di 'aletta ‘.'

L' abruzzese pennuccia è 'il culmine di un tetto a due spioventi', come a dire lo spartiacque del tetto. ${ }^{3}$ - Il napoletano pennata (e pennatella) è 'gronda di tegole o di legno sopra finestre o porte' e 'visiera del berretto'; il siciliano pinnata vale 'gronda, tettoia, tetto in luogo aperto, portico' (Traina), quindi la frase fari pinnata farsi il solecchio. ${ }^{4}$ Nell' uso toscano e letterario, pennato (già in Jacopone) è lo strumento agricolo tagliente e adunco per potare, che ha una specie di cresta nella parte superiore; e una sua varieta si disse o dice pennata. Il pinnatus -a dei vocabolarii latini non è che variante di pennatus, salvochè forse in un paio d' esempii dove si potrebbe discutere; ${ }^{5}$ ma a buon conto il pinnatus -a qual noi l' intendiamo sta in fondo cosl al 'potatoio' dell' Italia centrale (cfr. Zambaldi) come alla 'tettoia' della meridionale. - Un consimile rifrangimento di significati si ha in

1 Pei tanti penne ecc. in cambio di panne 'panno' cfr. il Littré s. panne.

2 In tal caso il pennon poco fa ricordato per 'penna della freccia' sarà fortuitamente omofono al 'pennone' e avrà un' origine a parte (penna).

${ }^{3}$ Non l' ha il Finamore, ma me lo dà il prof. Donato Forlani pel suo vernacolo di Sant' Apollinare (frazione di San Vito).

- A Trapani, mi assicura il prof. G. Luppino, pinnata è l' equipollente del nap. suppigno, di cui fra poco; e a Comiso (Modica), come un altro cortese $m$ ' avverte, é una specie di stalla a tre mura, col quarto lato aperto, e coperta di tetto.

5 Quello botanico, relativo a foglioline schierate di qua e di là del gambo, puó includer piuttosto l' idea di pinna; e così certo lo adottano $i$ botanici moderni (pinnato, fr. pinné e pinniforme). 
pinnetta, che in sardo è 'capanna', specialmente dei pastori o dei banditi; $e$ in siciliano è 'mensola del fondo della botte' (Traina), come l' abr. pennizze (plur.) è 'doghe laterali del fondo di botte o tino' (Finamore). - Lo Spano registra il logud. impinna 'a ridosso, dietro', ma avrebbe dovuto aggiungervi in pinneddu (*pinn- ello) 'in cima in cima'.' - Egli registra pure pinnacuzza 'muro a cresta che sporge'. -

Il molisano pinci (pinge), tegoli ed embrici, che mi restò finora un problema insoluto, credo oggi risolverlo postulando un *pinnici. Il suo singolare ('pinnicus) dovrebb' esser pinco (pinghe), ma è pincio (pinge), certo cosi foggiato sul plurale; il quale, sinonimo com 'è di 'tetto' $e$ indicando cose solitamente consociate, ricorre tanto più di frequente che non il singolare. L' abruzzese però risponde con entrambe le forme secondo i luoghi: pénge e penghe o pinghe (Finamore). ${ }^{2}$ Per la sincope e per altro, si ricordi che di quella stessa regione è Minco (Minghe) Domenico, la cui gutturale sonora, promossa dalla nasale, ha origine diversa dai Minghetti, Menghini, Minguzzi e sim. dell' Italia Superiore passati per la trafila di Meneg-. 3

Sotto ai pinci sta il suppigno, voce comune a tutto il Mezzogiorno continentale, che val 'soffitta', stanza a tetto più o meno inabitabile, ove piuttosto si ripongono cose diverse: a Bologna si chiama 'granaio', per esservisi in altri tempi riposto il grano. $\mathbf{C i}$ fa postulare un *subpinneum; e le carte latine di Puglia ci aiutano. Nel 'Chartularium Cupersanense' del rimpianto Morea (Montecassino 1893 ), al testo ipsa casa sicut est orriata ( $\mathrm{p} .6 \mathrm{r}$ ) trovo annotato che anc' oggi si dice da quelle parti orio (horreum) la soffitta più alta, da riporvi il grano o anche dormirvi, a differenza dal suppigno che è più basso. Al suppinno di un documento dell' a. 108I il M. annotando sembra ammettere che anche codesta forma

${ }^{1} \mathrm{Di}$ ciò m' avverte il collega Fadda.

${ }^{2}$ Gli Statuti municipali di Teramo latineggiano la nostra voce in pinsius, ed in pinzarius il 'faubricante di tegoli'. Cfr. abr. pingiare, tegolaia, topaia, casupola. - In qualche vernacolo (p. es. Telese) si ha pinciata, tetto.

' Il Pincio di Ruma, che ad altri parve forse connesso al 'pino' (cfr. Arch. Gl. X 312 n.), nel qual caso bisognerebbe postulare un di quei mostri accentuali per me incredibili, *pin(i)ceus, si sa che invece fu gia latino (Pincius, Pincianus), e prese nome da una famiglia cospicua nell' età imperiale, i Pincii (cfr. Huelsen, Röm. Mittheil., 1889, p. 269, cit. dalla contessa Lovatelli, in $N$. Antol. del $1^{\circ}$ maggio 1904, p. 68-9). Resterebbe quindi solo da vedere se il tosc. pinco 'pene' e 'scioccone', che (per eufemismo, secondo il Petrocchi) si cambia in pincio, e l' antiq. pinca, specie di cetriuolo, abbiano alcun legame col termine sannitico. Sebben questo potrebbe prestarsi ad applicazioni oscene, e benchè p. es. citrullo sia un napoletanismo ( $\mathrm{Caix}$, Studi di etim., p. 102), non es verosimile in questo caso la provenienza meridionale. Nemmeno è da pensare a sviluppo indipendente d'un'identica o consimile base, chè *pinnicus o*pinicus o *penicus (da pēnis) non avrebbe in toscano sofferto la sincope. E un problemuccio che forse si risolverà ricorrendo a lingue straniere, e qui non ci riguarda. 
esista oggi oltre quella in -igno, e avverte ad ogni modo che il nostro vocabolo, oltre che 'soffitta' può colà significare un edificio terreno, senz' altra copertura che il tetto, un capannone di ricovero pegli animali e pei lavoratori: il che ci ricorda il sardo pinnetta $\mathrm{e}$, più, il trapanese pinnata, veduti più sopra. Nel 'Codice diplomatico barese', vol. V (Bari 1 902) edito da F. Nitti di Vito, occorre suppinnum (bis a p. 31 a. I09I), suppinnulo (p. 4 a. 1075), casa suppinnala (ter, e suppennata: p. 52 a. 1099) con le frasi 'una casa orreata et una suppennata ... que casa et suppinnata'; e nel Glossario si rimanda al subpinna del vol. IV.1 Ed oggi i notai pugliesi volentieri scrivono la suppenna; dove è bello richiamare, malgrado l' applicazione diversa, lo sp. sopeña 'l' incavo sottostante a una rupe sporgente'; come, malgrado la stessa riserva, esso e il suppinnulo ci ritornano ai Soppenna, Suppinnula, Soppénnori, del Pieri.

Un *impinnea parrebbe ben adatto a spiegare la mpigna, che in tutto il Mezzogiorno, compresa la Sicilia, e non esclusa la Sardegna (impena, logud. impinna), si dice per 'tomaio', che insomma è la parte alta della scarpa, il convesso cuoio contrapposto alla suola. Anche il francese lo chiama empeigne (arc. empiegne, empangne), e gli risponde il Ducange con un impedia, che ha imbarazzato e fuorviato gli etimologi. $\mathrm{Ma}$ a crescer il buio o a far la luce sopravviene lo spagnuolo; il quale, oltre l'empeine impetiggine (origin. -ei-), e accanto all' empeine pube (che coll' it. pettignone, fr. penil, prov. penchenilh, pg. pentelho, risale a pecten, già classicamente usato, come $x \tau \varepsilon i_{\text {G, }}$ per 'pube'), ci ha l' empeine o peine, cui s'aggiunge o si sottintende del pie e che significa il 'tarso'.2 Questo può certo arieggiare il pettine, a causa delle cinque dita e delle ramificazioni ossee che le precedono. ${ }^{3}$ Orbene, la metonimia dalle parti del corpo alle rispettive coperture è ovvia, sia contrassegnata da formazione diminutiva, come in orecchino, corpetto, panciotto, colletto, polsino, pettino, sia senza ciò, come in collo, polso, petto, busto, vita, corpo (di camicia, o, in certe province, anche del vestito donnesco, e cfr. sp. en cuerpo 'senza mantellina'), dilo (di guanto), calcagno (di calza o scarpa), gamba (dei calzoni); che se il procedimento inverso è ben più raro, non però è inaudito, e a prova ne basti cintola. Dunque il fr. empeigne sarà davvero quel

1 Nella prima delle due opere (p. 95) a un testo (a. 1054) che dà pinna - pinnaculam (sic), la nota del Morea spiega 'parte superiore dell' edifizio', e sembra insinuare che la sia tuttora vivente in Puglia. Cfr. pinna o pigna, che gli pare indichi un rione di Monopoli (p. 296 n.); e un 'in portu pinne sancti Cataldi' nel volume barese (p. 206).

" La parte del pié, que está entre la caña de la pierna y el principio de los dedos", spiega l' Accademia; e lo avvertiamo onde non si cada in distinzioni troppo scientifiche fra 'tarso' e 'metatarso'.

8 Lo stesso 'tarso' valeva in greco anzitutto 'graticcio', e applicandosi, già nell' Iliade, a indicar la parte del piede che è tra il calcagno e le dita, mostra come l'immagine fondata sulla considerazione anatomica non ripugni anche alla comune intuizione. 
che già materialmente apparisce, un *impeltine, ed avrà preso le mosse preistoricamente da quel senso che nello spagnuolo è rimasto vivo e unico (il 'tomaio' vi è pala); ma nulla induce a credere che sia addirittura un antico spagnolismo, con senso oltrepassato. Come pure non è d'altra parte niente probabile che inversamente empeine sia un francesismo con senso alterato, e che empeigne, che poi sarebbe parso *impettine all' intuitiva spagnuola, sia invece, secondo un riservatissimo sospetto del Littré, un' altra applicazione della stessa voce germanica onde s' ebbe empan spanna; giacchè, se l' adulterazione dell' iniziale non è troppo strana, ed ha codesto così prossimo conforto, l' -eigne sarebbe sorprendente. Comunque sia, è manifesto che il nostro mpigna non è che un francesismo, come lo è il pg. empenha, che vale 'tomaio' e che dovrebbe sonare * empente se fosse indigeno. Sola singolarità è che Napoli e il Sannio non dicano mpegna, come e la loro fonetica e il modello francese vorrebbero, sicchè il loro - $t$ - debba spiegarsi o con la prevalenza della forma siculo-calabra, o, meglio, con la pretesa di clar al vocabolo la forma italianeggiante (cfr. it. gramigna di fronte al molis. gramegna, nap. grammegna). Taccio dell' Abruzzo perchè il plur. mpigne, che il Finamore dà per difettivo di singolare, ̀̀ colà del tutto in regola con la fonetica e con la mozione dei numeri; cosicchè, se talvolta vi si udisse il singolare e questo fosse con $i$, vi sarebbe stato trascinato dal plurale. Del resto anche in Sicilia ecc. l' $-i$ - è anorganico e dovuto alla prevalenza di questo suono nel relativo ambiente dialettale, e lo stesso è a dire suppergiù della Sardegna, dove anzi s' è visto che ha luogo un' oscillazione. Son tutte cose che rendon più manifesta l' origine esotica della voce, che ad ogni modo non ha che fare con pinna.

Ne so se ci abbia che fare l'abr. sannit. pennazza, col plur. pannázzerg (-ázzare, in Cremonese, Vocab. d. dial. agnonese, Agnone 1893), spiegato con 'palpebre' o con 'ciglia'. Mal si può definire se ia prima intuizione si volgesse aila convessa pelle palpebrale $O$ ai peli che la terminano, i quali ci sospingerebbero piuttosto a penna, come più decisamente $f a$ il calabro pinnulír ciglio (Scerbo, Sul dial. cal., Firenze 1886, p. I15).

Ma pennacchio non sarà proprio pinnaculum? ${ }^{\text {Che di solito }}$ ei sia di penne, e che ormai i parlanti pensino a queste, non toglie che '' idea che vi campeggia in modo quasi assoluto è quelia della cima, del cimiero; e quanto diverse potrebbero essere le sue vicende ideologiche se il punto di partenza fosser le penne, ce lo insinua piumaccio. Il fr. panache (talora fu scritto pennache), benchè gid se n' abbia es. del s. XV, e lo sp. penacho, pg. pennacho, non ci servono a nulla, poichè sono evidenti italianismi. Spennacchiare, benchè sorto presto (Petrarca ecc.), non è che un dispregiativo di spennare, e non ha veri rapporti col sostantivo, salvo forse nel-

1 Cfr. Körting, n. 7r 7 r.

Zeitechr. f. rom. Phil. XXVIII. 
l' averne un po' favorita la variante intensiva spennacchio, i cui primi esempii son del s. XVI. D' altra parte, che valore avrebbe qui il suffisso riferito a penna? Non diminutivo, come in orsacchio e sim., nè operativo, come in spauracchio e sim. Per l' uno non gioverebbe citare pennello, nato da un curioso intreccio; per l' altro, non mi par cosa tanto liscia il supporre questa base concettuale: "cio che impiuma l' elmo'. È preferibile un -aculum di tradizione latina e accennante a 'cima'; altrimenti la formazione più naturale sarebbe un *pennaceum, donde *pennaccio, che è riflesso sol nei sardi pinnazzu, -acciu. Quanto a pinnaculum, è bensì vero che non appartiene al fondo classico, e i primi suoi esempii son di Tertulliano e della Vulgata; ma è una regolare formazione sul tipo di tabernaculum, che è del latino aureo e pur esso non è appoggiato a un verbo realmente esistente, bensì a taberna. Così su pinna, in quanto vale cresta o culmine, un pinnaculum equipollente a 'fastigium'. Ed è vero che nella stessa latinità sacra fa capolino un pennaculum 'piccola penna', ma è di formazione meno regolare, e codesto senso non sarebbe, lo ripeto, il più adatto per istar a base di 'pennacchio'. Nè più calzante riuscirebbe il pennatulus di Tertulliano, che, a tacer d'altro, avrebbe dato piuttosto un aggettivo, $o$, se sostantivato, un nome non includente la nozione del culminare su un elmo o su un cappello. Tutto è possibile certo, e ci troviam davanti a uno dei casi dove più torna difficile fare il taglio netto tra la pinna e la penna, e dove puo essere illusorio appunto l' intuito che sembra sgombrare felicemente una volgare illusione. Alla fin fine il mazzo di penne non suole mettersi che in cima, ed il fatto materiale potrebbe a torto far parere che tale nozione sia essenziale e originaria. Ma nonostante questa riserva, la nostra equazione può esser posta con sufficiente franchezza, senza neppur discendere a chieder aiuto al pennacchio architettonico 'pietra su cui posano gli spigoli delle volte': dove la mancanza di ragguagli storici e tecnici ci lascia dubbii se non si tratti d' una semplice metafora. Ma non trasandiamo di rifarci al Pennácchiore del Pieri: ove l'-ore non può essere che un mascherato plurale del tipo prátora ecc., e ci fa anzi nascer il dubbio se anche Pennora, Pennori, Soppénnori e, più ancora, Trapénnori (se è intra- anzichè ultra-) non debbano spettare alla medesima genia, per quanto - or- $=-u l$ - sia non meno aspettabile in quell' ambiente (cfr. Arch. Gl. XII 117 ).

Una buona consistenza ha pure la vicenda semidotta del vocabolo: it. pinnacolo (ant. pinacolo, pennacolo), fr. pinacle, sp. pg. pináculo. E molta vita esso ebbe nella latinità medievale (ove finanche pinnaculabit), anzi si può dir che giovò a sorreggere, tra le persone colte, il sostantivo fondamentale, rendendone più chiara e consapevole la distinzione dall' insidioso gemello. Così si trovano abbinati pinna e pinnaculum, o pigna e pignaculum (Ducange); e quel pinnaculam di Puglia, se non è mera svista, accennerebbe a impeti di maggiore compenetrazione. 
Altre vicende dotte o semidotte: it. pinna dei pesci, o del naso, o specie di conchiglia (a che forse s' attiene il sd. pinnadellu, -eddu, giavazzo, ambra, margheritina), pinnula, -ola, duplice laminetta di un certo strumento ottico; sp. pina merlo di muro, limite o pietra terminale di figura conica, ciascuno dei quattro pezzi arcuati che uniti formano la circonferenza della ruota (it. quarto), pinula (la pinnola diottrica anzidetta); pg. pina quarto di ruota, pinnula pinnola; fr. pinne (ant.) dei pesci, pinne marine (conchiglia $\pi i v v \alpha$ ), con pinnier (il mollusco che l' abita) ecc.

In conclusione, nella dura lotta per la sua esistenza, il pinna, con suoi vecchi o nuovi derivati, riuscì a tenersi più o meno vivo, e nella tradizion letteraria e nell' ingenua tradizione orale. Scarsamente vi riusci in Francia, ove poi ebbe a competere non solo col penna, ma altresi, come questo stesso, con pannus, ${ }^{*}$ panna; moltissimo in ispagnuolo, dove quasi trionfo dell' incomodo gemello; un po' meno in portoghese; non molto meno in italiano, nel meridionale in ispecie. Non sarà quindi audace postular nella latinità italo-spagnuola un *impinnare, donde, in vicenda semidotta, lo sp. pg. empinar, e per trafila popolare, o in popolare abbigliamento (cfr. discepolo e sim.) il nostro impennare. Di questo non si hanno esempii che ci riportino addirittura alla lingua di Dante o giù di lì, ma a dissuadere ch' ei si riduca a uno spagnolismo, come anche il significato più circoscritto fra noi e più tecnico potrebbe far sospettare, basti avvertire che in età più antica prevalse rimpennare.

La Crusca lo dà con esempio da un 'Libro di motti', vecchio manoscritto smarrito da un pezzo, e alla cortesia dell' amico Del Lungo devo un istruttivo confronto fra le successive edizioni del Vocabolario della Crusca. La quale nella prima stampa (1612) registra rimpennare senz' altro, nella seconda (1623) aggiunge 'Oggi impennare', nella terza (I69I) scrive 'Oggi si dice comunemente impennare', nella quarıa (1729-38) scrive 'che più comunemente oggi diciamo impennare'. Il Del Lungo conchiude: „Col Seicento insomma il rimpennare cavallino è morto, e trionfa l' impennare odierno". Più giusto dunque è sospettare che l' influsso spagnuolo abbia contribuito a dar la prevalenza al termine senza $r-;$ e del resto, quando pure lo stesso rimpennare risultasse spagnolismo, rulla ne soffrirebbe la sostanza della nostra tesi. 'L' inpinnare, -ai, che lo Spano registra nella parte sarda del suo sconnesso volume, più difficilmente potrebb' essere, stante la nasale doppia, uno spagnolismo, e più facilmente un italianismo; ma nulla vieta che sia indigeno, e faccia testimonianza di bella continuità per tutta la latinità meridionale. L'impinnire, -iri, che pone nella parte italiana a riscontro dell' impennare in entrambi i sensi („Pienare de pinnas. Alzaresi derettu de su caddu“), o ¿̀ una variante secondaria che non isconcerta nulla, $o \grave{\dot{y}}$ un italianismo in quanto la parificazione morfologica dei due verbi potè doversi alla parità materiale di essi due nella lingua letteraria. Ma c 'è 
il caso che la verita sia che -are, -ai, si dica pei cavalli, e -ire, $-i r i$, delle piume. 1

1 Il fr. se cabrer, ideologicamente tanto piano, per esser la capra tanto svelta ad arrampicarsi, cosi da parere che ogni altro quadrupede nell' impennarsi la imiti, foneticamente è bisognevole di spiegazione. Gli accenni del Diez al neoprov. cabrd, e del Littré allo sp. cabra, sono indeterminati; e con egual ragione, almeno in apparenza, si potrebbe invocare il sost. cabre della Francia settentrionale. Altre voci congeneri, o semidotte o dialettali o straniere, sono: cabre (ordigno marinaresco da alzar pesi, cavalletto dei tessitori), cabrz (sp. cabrito), cabrillon (cacio del Lionese o dell' Alvernia), cabriole ant. capriole (ital.), cabrioler ant. caprioler, cabriolet, cabrioleur, cabrion, cabron. Ora cabrer (dal s. XVI) viene dal nord o dal sud? da un vernacolo o dall' estero? Parrebbe dalla Spagna, la quale oltre i sinonimi già studiati possiede encabritarse. Vero è che in tal caso si aspetterebbe che il francese o lo ripetesse meccanicamente con un *encabriter, o se lo accomodasse più consapevolmente $a$ un *enchevretter; ma, se dai vernacoli non verranno altre indicazioni, bisognerà pur credere a un rifacimento mezzo grosso e mezzo fino del termine spagnuolo, tanto più che dialetti intermedii avran potuto contribuirvi con fasi digradanti. Certo che l' origine spagnuola è raccomandata anche da ragioni storiche. Il Nigra ha la bontà di scrivermi: "I vocaboli relativi alla cavallerizza non vengono dalla Francia occidentale o del nord-ovest, ma dall' una o dall' altra delle due penisole, dove quell' arte fu insegnata da trattati speciali. La Spagna introdusse la cavallerizza a Napoli [qui infatti due strade han per nome Cavallerizza] e di là nel resto d' Italia e poi in Francia, ed ora è soltanto conservata a Vienna (parlo della vecchia arte)". - Intanto queste utili avvertenze mi conducono a rendermi conto di cavalleriezza appunto e cavallerizzo e di rubizzo, che ora mi paion spagnolismi tutti, benchè il primo si trovi gia in Andrea da Barberino (s. XIV-V): del secondo il più antico esempio è del Buonarroti, e il terzo incomincia coi Canti Carnascialeschi e col Machia. velli. Lo sp. caballeriza significa soltanto il locale, ovvero la truppa dei cavalli e dei famigli, non già l' arte in sè (che è manejo), mentre in italiano può significare anche l' arte; ma l' ampliamento del significato non nuoce alla tesi, che negl' imprestiti suol proprio aver luogo o la restrizione o l' ampliamento. Qui poi è il suffisso che decide: ovvio nello spagnuolo (p. es. hechizo), eteroclito fra noi (fittizio non *fittizzo). Solo esempio ne parrebbe rubizzo, se davvero includesse l' idea di 'rubicondo' e si potesse postulargli un $*_{r}$ ŭ bitius. Ma che valore ha codesta etimologia?! Certo, lo zz e qui sordo, e la forma con $z z$ sonoro ammessa come secondaria dal Petrocchi, non legittimerebbe un ${ }^{*}$ rubidius da rŭbidus, o dal rūbidus = ruvido di cui disserto lo Schuchardt ('Romanische Etymologieen', I, 20 sgg.), chè ne sarebbe venuto *ro- o ruvezzo o -eggio; e quella forma secondaria è una delle tante degenerazioni delle sibilanti, specialmente in parole antiquate o esotiche, secondo ho mostrato altrove. Rubbizso dunque è semplicemente lo sp. roblizo gagliardo; e bene sta che un aggettivo, di cui i lessici italiani avvertono che si applica in ispecie a vecchi robusti, significhi in origine quasi *rovereccio. $\mathrm{Ne}$ chi pretendesse indigeni cotali -izza, -izzo potrebbe appellarsi a stravizzo per stravizio, voci problematiche esse stesse. Vengon sù nel Rinascimento; e la prima forma prevale nell' indicar il famoso pranzo della Crusca al qual teneva dietro la 'cicalata', mentre la seconda prevale nel senso più ordinario, senza che però possa stabilirsi una rigorosa sinonimia. Taluno l' interpretó come 'esterno vizio', altri come un *extrabibitio; a cui si potrebbe forse surrogar *transbibitio (cfr. straporto). Ma altri lo trasse dallo slavoz draviza (cfr. Tomm a seo, Sinonimi); e forse è questa la vera origine, e il 'vizio' c' entrò poi di straforo, per etimologia popolare moralizzante. In ogni caso non si tratterebbe del suffisso che a noi preme, e la coesistenza stessa delle due varianti ci porta gia in un altro campo. - In francese, la cavallerizza e la sua arte non è che manège, e il Littré vi riconobbe un italianismo. Maneggio infatti gia in Davanzati (il relativo verbo in Cellini) ed ha un significato 
altrettanto esteso, onde pare l'equivalente indigeno della spagnolesca cavallerizza. Anzi lo sp. manejo, che indica soltanto l' arte, sarà pur esso un italianismo, benchè in astratto sia possibile la sua diretta coniazione in Ispagna. Certo vi sentì l' italianismo un cinquecentista spagnuolo, che scrisse: „De la lengua italiana deseo poderme aprovechar por la lengua castellana destos vocablos, facilitar, fantasia en la sinificacion que lo tomais acá; aspirar por tener ojo, como quien dice: Cada cardenal aspira al Papado; dinar, entretener, discurrir, discurso, manejar y manejo, deseñar y deseño, ingeniar por inventar con el ingenio, servidumbre, novela y novelar, comodo y incómodo, commodidad, solacio, martelo (porque no parezca que es lo mesmo que zelos), pedante y asasinar" (dal vol. "Origenes de la lengua española compuestos por varios autores, recogidos por d. Gregorio Mayans y Siscar', Madrid, 1873, p. 104). - Tornando ancora un momento agl' -izzo, mi risovviene di novizzo -a. I lessici lo danno come una rara variante del più comune novizio; e in particolare per novizza asseriscono averlo usato il Caro per 'sposa', al modo veneziano. Ma, come mi avverte il Del Lungo, non il Caro, bensì il Casa l' adoprò: in una letterá da Venezia, del 12 marzo 1545, e parlando di nozze venete. La lettera è fra quelle a Carlo Gualteruzzi pubblicate dal Rezzi (Imola 1824), e il passo (p. 12) dice: , La sig. Agnola si è contentata di pagar la dote come il sig. Ridolfo ha voluto, ed hanno mostro la sig. novizza all' Arme, che se ne è contentato assai, e la sig. Agnola vi ringrazia". Così il venetismo del sostantivo femminile è fuor di questione. Ma anche l'aggettivo e il maschile avranno la medesima origine. Certo, dice il Del Lungo, nè sono nè furono mai toscani. Infatti i lessici non ne sanno dare alcun esempio classico, e ogni Italiano vi sente un non so che di vezzoso, fuorchè $i$ veneti che l'hanno nel loro vernacolo; nel quale tuttavia si dice patrizio, non *patrizzo, cosicchè novizzo par come l' equivalente di un *noziccio, a guisa di pastizzo = tosc. pasticcio. Il classico novitius, -icius, $s$ continua dappertutto altrove in regolare vicenda semidotta: it. novizio, sp. novicio, fr. novice (fin dal s. XV), pg. noviço (francesismo?); ma il riflesso veneto sembra essere entrato nel filone particolare degl' -iccio.

\section{F. D' Ovidio.}

